\title{
Enhancing AODV routing protocol to support QoS
}

\author{
Ara Miran ${ }^{1}$, Govand Kadir ${ }^{2}$ \\ Assistant Lecturer, Department of Information Technology, College of Engineering and Computer Science, \\ Lebanese French University, Erbil, Kurdistan Region, Iraq, ara.zozan@ @lfu.edu.krd \\ Assistant Professor. Department of Computer Science, Collage of Computer Science and Engineering ,University \\ of Kurdistan Hawler, Erbil, Kurdistan Region, Iraq, g.kadir@ukh.edu.krd
}

\begin{abstract}
Mobile Ad-hoc Networks MANET support for Quality of Service (QoS) is bonded by the capability of the routing protocol to select nodes that can provide the necessary resources that meet the QoS requirement. MANET is formed from wireless devices (nodes) that have limited resources and lacks any central management entity. Therefore, nodes inside the network have to exchange extra information to gain knowledge of other nodes capability inside the network, which in result consumes nodes limited resource. This paper proposes an enhancement to reactive routing protocol such as (Ad-Hoc ON Demand Distance Vector (AODV), by adding extra information about nodes processing delay to the routing messages and share it with the source of data that requested a route. Then the source node uses this information with the hop count gathered through the routing process to send the data that requires less delay through a path that combines the shortest path with minimum delay. Simulation results conducted using riverbed simulation software shows an improvement in the packet delivery ratio PDR and End-to-End delay for Minimum Delay Ad-Hoc ON Demand Distance Vector) (MD-AODV).
\end{abstract}

Key words: AODV, MANET, MD-AODV, and min_delay.

\section{INTRODUCTION}

MANET is the networking technology that suits the mobility and freedom of wireless devices like Smart-Phones. People want to join and leave networks as they want and do not require sophisticated processes applied by service providers and. It can be a good alternative when these providers are down during disasters or war. The advancement in wireless devices and the increase of data exchange speed, real time applications become a central source for communication among people.

Data exchange in MANET requires routes that it should be established before transmission and nodes participating in formation of the network have to cooperate and use some of its resources to forward data packets. For this purpose, MANET uses different type of routing protocols that can be classified in different ways.

The main classification relies on the route discovery policy. Proactive or static routing protocols require routes to be established between all nodes during the formation of the network as in (OLSR and DSDV). Routing tables used to store paths to all nodes inside the network and they are updated periodically. These routing protocols have the advantage of route availability when needed and the disadvantage saw in large networks as it causes overhead and consume resources. While reactive or dynamic routing protocols establishes path when any node have data want to send as in (AODV and DSR). The advantage of this type is the small size of routing table, as nodes do not require storing information about whole network the disadvantage comes from the delay time needed for route discovery and the delay caused by link break and repair process that leads to further packet retransmission. Combinations of both previous types create a hybrid type of protocols to reduce overhead caused by the two different types. This type usually suits large network in which it is divided to smaller groups called (clusters, zones....etc.) inter-group communication conducted in a proactive manner while inner-group communication conducted reactively, the disadvantage of these protocols come from the larger memory requirement caused by storing higher-level topological information, and the significant overlap in routeing zones (Raheja \& Maakar, 2014).

Generally, nodes in MANET have limited information about others beyond transmission range. On the other hand, QoS provisioning require routes that can guarantee services and for this needs to share nodes capability with others inside the discovered path. Sharing knowledge about other node capabilities require the exchange of extra messages that causes overhead and consume resource. Therefore, it is important to use routing protocols that have limited support to QoS. Accordingly, routing protocols messages have to be enhanced to carry extra information about QoS parameters and shared it among nodes residing inside the path (Kadir, et al., 2016). 
The proposed enhancement modifies the routing protocol and the shortest path concept that relies on number of hops and adds the processing delay of the nodes. This process requires the nodes inside the path to share the accumulative delay parameters during the RREP process. Source node then selects a path that combines minimum delay caused and least number of hops to select the best path to send the data.

The rest of this paper organized as follows: Section two provides a review of the research works conducted in this area. Section three introduces the enhanced AODV routing protocol, while Section four simulate the MD-AODV routing protocol using riverbed simulator, then Section five examine the results obtained from the simulation of different scenarios. Finally, we conclude the improvement obtained in comparison to the original AODV routing protocol in section VI.

\section{LITERATURE REVIEW}

MANET routing protocol improvement motivated researchers to explore different ways to enhance its support to QoS. In this section, we explore some of these efforts and analyze the results.

A Research study evaluated different reactive routing protocol support to QoS, and for this purpose AODV, DSR and TORA protocols considered. The review studies many different parameters such as (route discovery, packet delivery and delay ).The result show different performance for each protocol, but overall AODV outperforms the other protocols regarding media access delay, load, delay, data drop retry, and retransmission attempts (Ali \& Kulkarni, 2017).

In another research, the performance of AODV and the QoS support was examined in a large MANET. The network was divided to chunks and each chunk has a (CHG) Cluster Head Gateway that spread the load among multiple hosts in the cluster using (MCHG) MANET Cluster Head Gateway (Bagwari, et al., 2012). Results from different performance parameters demonstrate that AODV have better response in term of throughput and End-to-End delay requirements and less packet drops.

Meanwhile, a different research enhanced AODV routing protocol using a priority mechanism by sharing information among nodes inside the transmission path in the network. AOMDV proposed to enhance the QoS by estimating the available bandwidth in IEEE 802.11 based MANET with different connection preferences based on the data rate. The protocol passes user requirement for specific data rate from higher layer, and then nodes compare the information with a threshold and process it accordingly. This information helps toward achieving better QoS (Jayabarathan, et al., 2015). The proposed work is promising although little details provided about the mechanism used to pass information and the threshold update process.

Another research enhances resource consumption by reducing the unnecessary signaling and prevents sessions that cannot meet QoS requirement (Renesse, et al., 2004). The proposed solution consider the immediate bandwidth information of nodes in the network using hello messages and estimated the impact on both the AODV and QoS-AODV routing protocols. We believe that sharing information between nodes helps toward establishing paths that can meet QoS requirements but with a careful consideration for overhead that consume resources.

Load-Balancing DSR Based QoS Routing Protocol in MANETs (RTLB-DSR) is another enhanced routing protocol that adds some QoS parameters to DSR protocol to achieve adaptability and strengthen the protocol (Maleki, et al., 2014). Various policies applied to obtain flexibility for load balancing. The promising results obtained from different scenarios prove that adding some of QoS metrics to routing protocols will improve the performance and a similar approach was adopted in our work.

A different concept in another research tries to reduce the overhead caused by link break of paths due to mobility, which in return requires constant route discovery attempts. The proposed method tries to minimize the broadcast messages. This study aims to determine the possibility to postpone the calculation and likelihood of Probabilistic Rebroadcast (PR) protocol by reducing the routing overhead in highly dynamic network. PR protocol needs Hello packets to gain information about neighbors, and include the neighbors list in the RREQ packet. The aim is to use some techniques to minimize the overhead of Hello packets and select certain neighbors from the RREQ packet list (Suradkar \& Surve, 2014). The simulation result show an improvement in End-to-End delay. We used a similar concept in using route discovery packets to share information.

One more work improves AODV routing protocol to select a path with high energy that will enhance the path life and prevent link breakage caused by nodes running out of battery (Naanani, et al., 2014). The proposed work introduces a formula to control the packet residence time inside a queue and in this way the energy consumption for processing packets get reduced and nodes lifetime is enhanced and in return we will have paths that last longer and prevent link breakage.

\section{PROPOSED MD-AODV DESIGN}

The route discovery process in the original AODV routing Protocol consists of the following two processes:

\section{Route Request process RREQ:}

- Starts with source node that have data to transmit which creates a RREQ message and broadcast it to neighboring nodes in transmission range as in Fig.1.

- Every node receives the RREQ checks for any previous valid path to the destination.

- If found then sends a RREP message back to the source, and sends a gratitude RREP message to 
destination telling it about the route request.

- Otherwise, if no route found then rebroadcast the message to its neighbors.

- This process continues until the packet reaches the destination.

\section{Route Reply process RREP:}

- Once arrived destination node creates RREP message and forward it back through the node that it sent the RREQ as seen in Fig. 2.

- This process continue every node updates the message and forward it to previous node until it reaches back to the source

- Once the source receives the RREP, it starts to send data immediately.

The proposed modification to AODV routing protocol consists of modification of the RREP process and source node, route selection. Therefore the RREQ process executed in the same manner as in normal AODV. While the RREP process, contains the following change:

1- A 32 bit filed added to the RREP message to store the accumulative value of the Minimum processing delay.

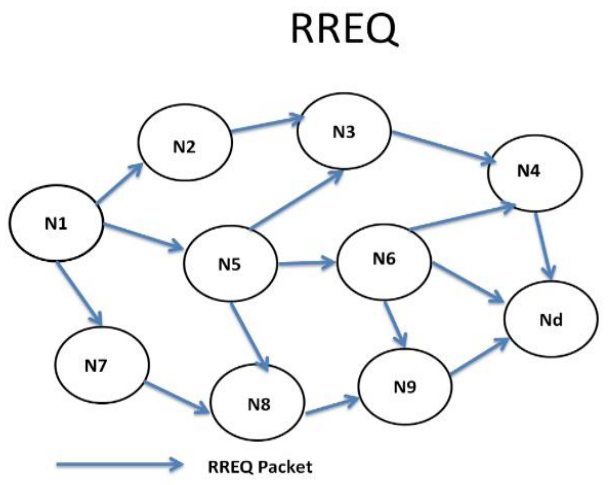

Figure 1: RREQ process in AODV routing protocol

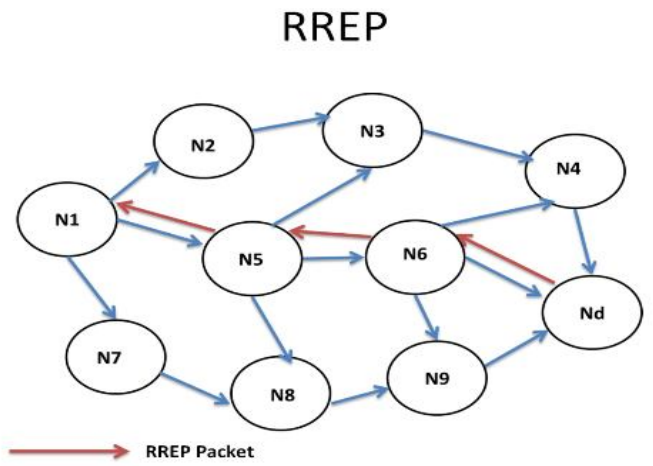

Figure 2: RREP process in AODV routing protocol

2- Starting with the destination every node processes the RREP message insert its own processing delay and forward back the RREP message as in Figure 3.

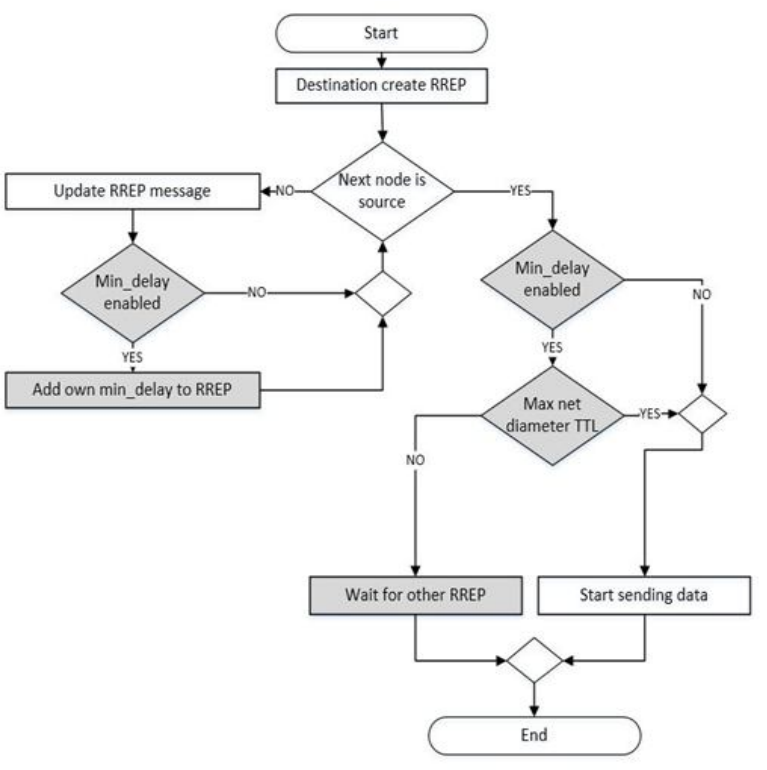

Figure 3: The modification to RREP process in AODV routing protocol

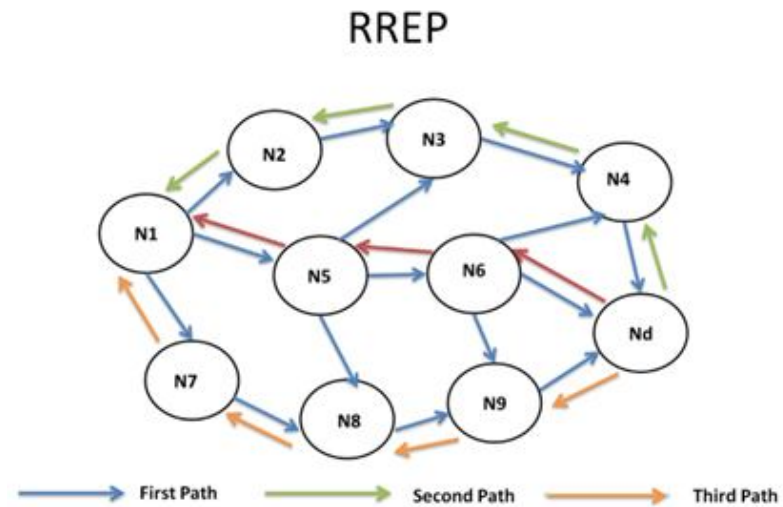

Figure 4: MD-AODV routing protocol with RREP from several paths

3- When the source receives the RREP message it delays the data transmission to allow other RREP message to arrive until maximum net diameter time expires as seen in Figure 4.

4- Then the source compares the minimum delay of each path and the no of hops to select the path. In which is used for data transmission.

5- A reference for other paths stored to be used in case of congestion or link breakage.

\section{Algorithm Simulation}

This section demonstrates the implementation of MD-AODV into riverbed simulation software, in order to compare the performance with the original AODV routing algorithm. For this purpose, several simulation scenarios created to reflect different situations with various performance metrics. 
- Data Transmission Rate: data transmission depends on bandwidth we use, level of noise, and the signal. Mobile nodes feature different data transmission rates that lead to variable transmission ranges, these differences affect the performance of the routing protocol as lower data rate have higher transmission range (Sylvia, et al., 2013).

- Node mobility: represents the movement of the node in vicinity inside the network. This is examined through parameters like trajectory, distance, and node's speed. These parameters influence network performance characteristics like throughput and PDR (Hrudya, et al., 2013).

The comparison of the two version of AODV routing protocols is conducted using the following performance metrics:

- Route Discovery Time (RDT): represents the round trip time required for route discovery, which consists of the time required for the RREQ to reach the destination, and the RREP message to come back to the source (Khalid \& Ali, 2017).

- Packet retransmission (PR): "Represents the total number of retransmission attempts by the nodes in the network to deliver the packet successfully. Packet retransmission occurs because of link break that leads to packet drop because of node's processing delay.

- End-to-End Delay: used to measure the delay encountered for the transmitted data between the source and destination.

A. Scenario-I: The scenario consists of 20 MANET nodes placed randomly in an area of $(2000 \times 2000 \mathrm{~m})$ as in Figure 5. This is to provide enough room to accommodate a typical path consist of several hops inside the network, bearing in mind that the maximum transmission distance between any two wireless devices is (300m) (Lu \& Yang, 2012). Furthermore, two nodes (2 and 20) exchange multimedia streaming data in opposite direction. Random delay was assigned to nodes inside the network that will be considered by MD-AODV during route discovery. Several simulations executed using ascending data rates values to all nodes.

B. Scenario-II: The previous scenario modified and nodes were made mobile with random trajectory (movement range $300 \times 300 \mathrm{~m}$ ) to allow nodes to transmit to maximum transmission range. The overall characteristics of the scenarios see in Table 1.

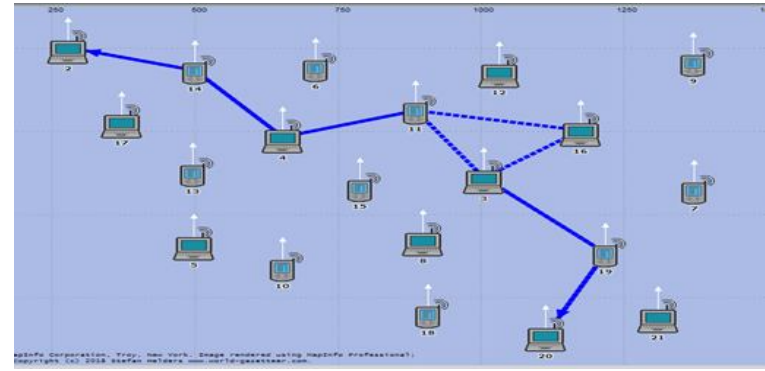

Figure 5: Scenario-I, MANET nodes distributed randomly.

Table 1: simulation scenario parameters

\begin{tabular}{|l|l|}
\hline Parameter & Value \\
\hline Trajectory & $\begin{array}{l}\text { VECTOR (Movement } \\
\text { range: 300m * 300m) }\end{array}$ \\
\hline Data rate (MBPS) & $6,9,12,18$ and 48 \\
\hline $\begin{array}{l}\text { Packet interval time } \\
\text { variance }\end{array}$ & $\mathrm{msec}$ \\
\hline Node traversal Time & $.04 \mathrm{sec}$ \\
\hline $\begin{array}{l}\text { Packet reception power } \\
\text { threshold }\end{array}$ & $82.65 \mathrm{dBm}$ \\
\hline Transmission power & $.0005 \mathrm{Watt}$ \\
\hline Active route timeout & $\mathrm{sec}$ \\
\hline Buffer timeout & $\mathrm{sec}$ \\
\hline Traffic mix & $.03 \mathrm{~GB}$, all explicit \\
\hline Simulation Duration & 5 minutes \\
\hline
\end{tabular}

\section{Results and Analysis}

In this section the simulation results for both scenarios is presented and analyzed

\section{Scenario-I (Fixed nodes)}

A. Route Discovery Time: The simulation result shows a path was established for both AODV and MD-AODV as in Figure 6. The RDT for MD-AODV took $0.1 \mathrm{msec}$ more on average in comparison to AODV. Obviously because of MD-AODV route discovery process does not end with first RREP packet arrival, instead it waits for RREP from other paths to arrive that requires extra time.

B. End-to-End Delay: The result shows that both protocols have a similar delay for $1 \mathrm{mbps}$ but normal AODV delay increases rapidly with data rate. This is because the data transmission goes in both directions an increase in data rate leads to process packets faster and if the nodes delay is large then packets dropped and retransmission is required, which in return accumulatively increases the End-to-End delay (Liu \& Chlamtac, 2004). Therefore we notice when MD-AODV consider nodes with less delay we 
obtained an average $3.5 \mathrm{msec}$ less delay as seen in Figure 7.

C. Packet Retransmission: During transmission routing error occurs as a result of link break or congestion, in which route repair starts leading to packet drop and requires retransmission. Simulation results show that both protocols gradually have an increase in the packet retransmission, which is due to increase in the data rate as previously explained. However, we notice that MD-AODV have an average of 50 packets for deferent data rates while the normal AODV have 55 as in Figure 8.

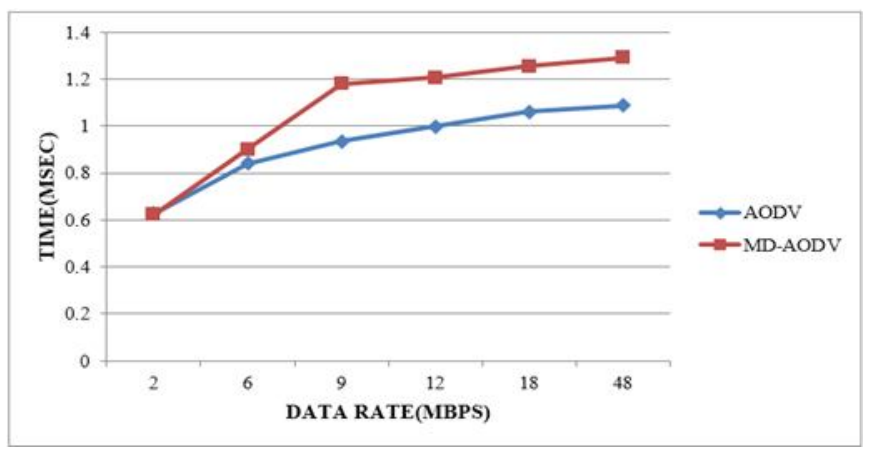

Figure 6: Scenario-I (Fixed nodes), Route Discovery Time.

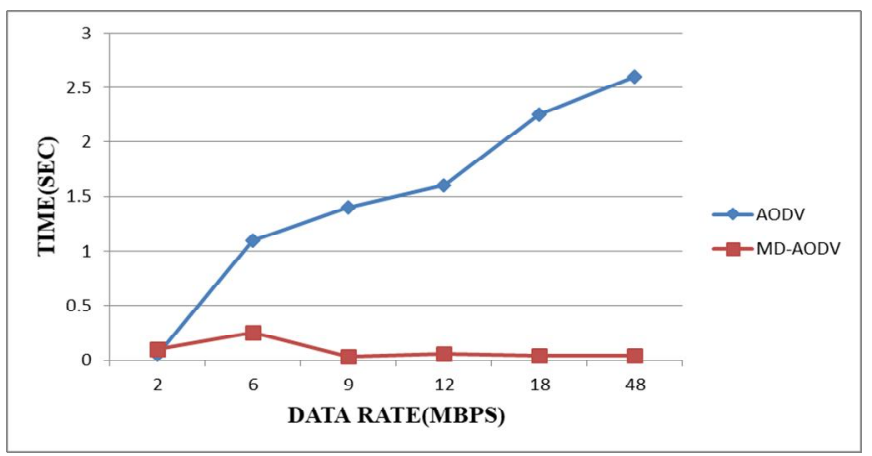

Figure 7: Scenario-I (Fixed nodes) End to End delay.

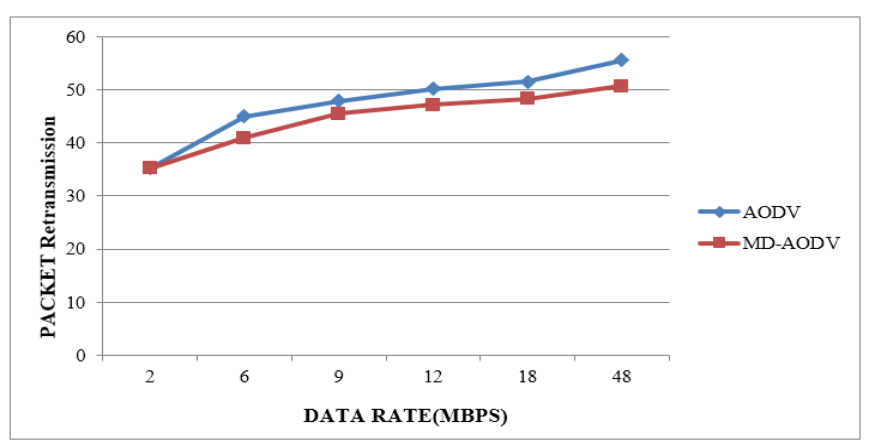

Figure 8: Scenario-I (Fixed nodes), Packet Retransmission.

This is another indication that a path with less delay causes fewer packets to get dropped and retransmitted again.

D. Packet Delivery Ratio: The PDR for both protocols shows an increase of $0.1 \mathrm{msec}$ on average in favorite of MD-AODV, because of the enhancement avoids paths that contain nodes with high processing delay, which in return leads more packet drop that in result leads to less PDR as seen in Figure 9.

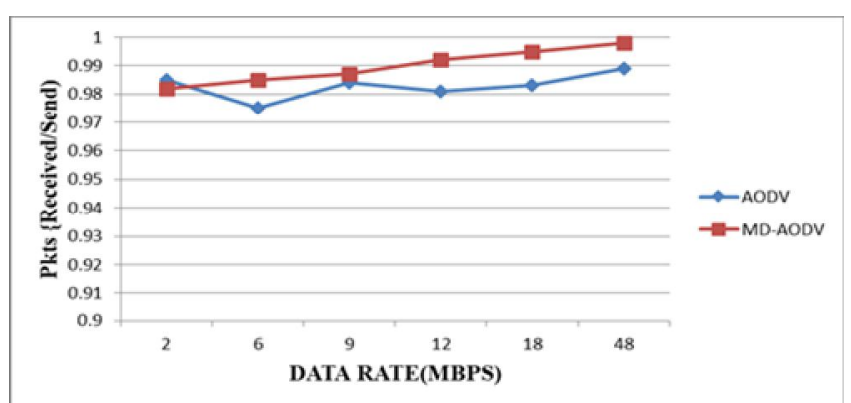

Figure 9: Scenario-I (Fixed nodes), Packet Delivery Ratio

\section{Scenario-II (Mobile nodes)}

1- Route Discovery Time: we notice from the simulation result that the RDT pattern is similar to previous scenario as in Figure 10. The average RDT for AODV is $1.5 \mathrm{msec}$ while for MD-AODV is $2.5 \mathrm{msec}$ on average. This time the differences between the two protocols are increased to $1 \mathrm{msec}$ or $0.9 \mathrm{msec}$ more for MD-AODV.

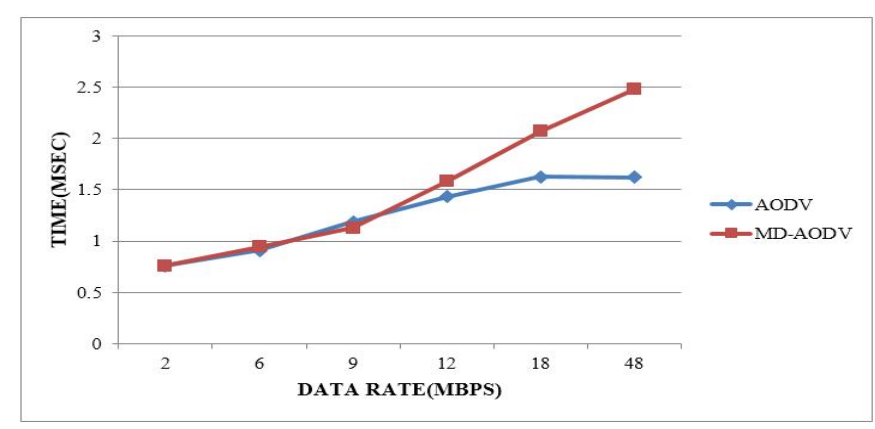

Figure 10: Scenario-II (Mobile nodes), Route discovery Time.

This is due to the mobility of the nodes which forces the source to take longer time to select a path and start transmitting the data.

2- End-to-End Delay: The simulation result shows a similar impact for nodes mobility on the End-to-End delay for both AODV versions with an increase of approximate $0.5 \mathrm{msec}$ on average as seen in Figure11. Furthermore, and for the same reason we notice that the delay increases for MD-AODV this time increase with data rate. 


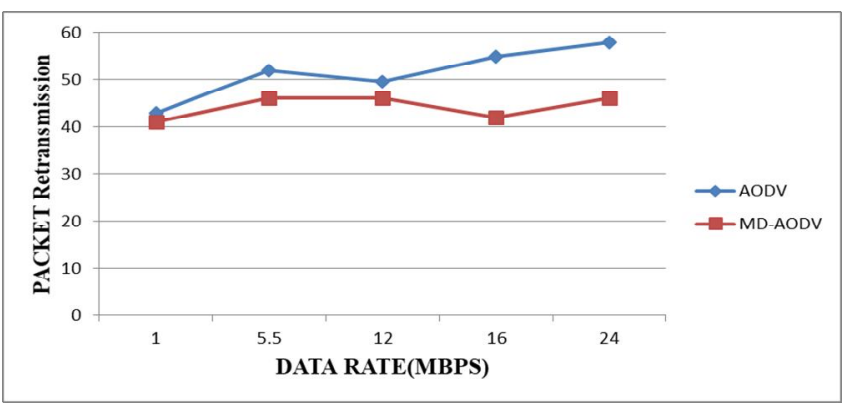

Figure 11: Scenario-II (Mobile nodes), End-to-End Delay

3- Packet Retransmission: unlike the previous scenario, the PR results of this simulation shows that the packet retransmission pattern varies for different data rates, this is due to the movement of the nodes as in Figure 12. However, we see similarity in the advantage of MD-AODV of 7.28 packets on average. Furthermore, different results changed when the simulation was repeated several times but the pattern and advantage of MD-AODV stayed the same on average.

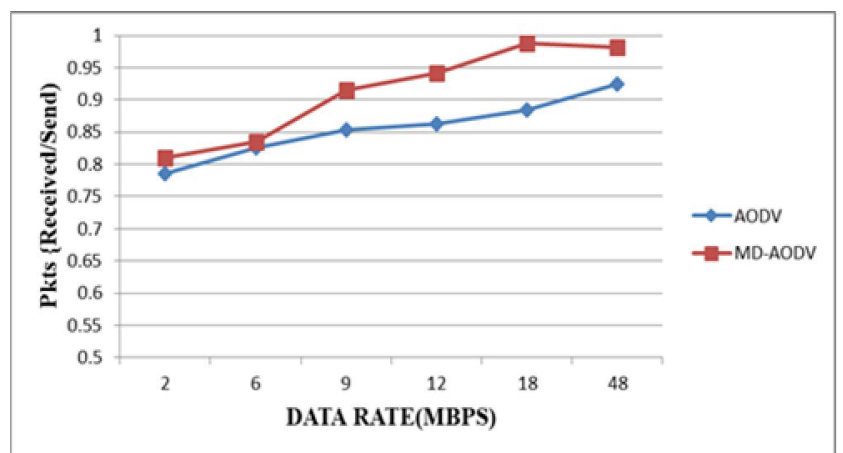

Figure 12: Scenario-II, Packet Retransmission

4- Packet Delivery Ratio: Simulation result shows a reduction in PDR which is due to packets drops occurs due to nodes movement, but MD-AODV still have the advantage of 0.912 to 0.855 for AODV because of node processing delay.

\section{CONCLUSION}

This research work presented in this paper shows that routing protocol can be improved further by formulating a mechanism to help nodes gain further knowledge of others inside the network. Without exchanging further message and extra processing burden on nodes. The primary challenge was to enhance the routing protocol but I always kept an eye on avoiding any alteration to the well-designed routing protocol and trying the best not to cause extra overhead that prevents the enhancement from achieving its goal. Overall, we can conclude that simulation for MD-AODV shows the correct implementation of the algorithm and the performance metric results proves the success of the implementation. The simulation result also shows previous two that MD-AODV will cause a slight increase in route discovery. However, it leads to a better End-to-End delay during data transmission.

\section{REFERENCES}

1- Ali, A. K. S. \& Kulkarni, U. V., 2017. Comparing and Analyzing Reactive Routing Protocols (AODV, DSR and TORA) in QoS of MANET. 2017 IEEE 7th International Advance Computing Conference (IACC), pp. 345-348. https://doi.org/10.1109/IACC.2017.0081

2-Bagwari, A., Jee, R., Joshi, P. \& Bisht, S., 2012. Performance of AODV Routing Protocol with Increasing the MANET Nodes and Its Effects on QoS of Mobile Ad Hoc Networks. s.l., s.n., pp. 320-324. https://doi.org/10.1109/CSNT.2012.76

3- Hrudya, K. P., Gupta, P. \& Kumar, B., 2013. Impact of mobility on different routing approaches in manets. International Journal of Computer Applications, Volume 67. https://doi.org/10.5120/11535-7296

4- Jayabarathan, J. K., Sivanantharaja, A. \& Robinson, S., 2015. Quality of service enhancement in MANET using priority aware mechanism in AOMDV protocol. s.l., s.n., pp. 1-5. https://doi.org/10.1109/UPCON.2015.7456735

5-Kadir, G., Kuseler, T. \& Lami, I. A., 2016. SMPR: A Smartphone Based MANET Using Prime Numbers to Enhance the Network-nodes Reachability and Security of Routing Protocols. IJ Network Security, Volume 18, pp. 579-589.

6- Khalid, A.-B. \& Ali, A., 2017. Multimedia Services and Applications in Mission Critical Communication Systems. First ed. s.1.:IGI Global.

7-Liu,J. J.-N. \& Chlamtac, I., 2004. Mobile ad hoc networking with a view of $4 \mathrm{G}$ wireless: Imperatives and challenges. Mobile ad hoc networking, Volume 1, pp. $1-45$. https://doi.org/10.1002/0471656895.ch1

8-Lu, Z. \& Yang, H., 2012. Unlocking the power of OPNET modeler. s.l.:Cambridge University Press.

9-Maleki, H., Kargahi, M. \& Jabbehdari, S., 2014. RTLB-DSR: A load-balancing DSR based QoS routing protocol in MANETs. s.1., s.n., pp. 728-735. https://doi.org/10.1109/ICCKE.2014.6993411

10- Naanani, H., Mouncif, P. H. \& Rachik, P. M., 2014. Improved AODV Routing Protocol for MANETs. International Journal of Engineering Research \& Technology (IJERT), Volume 3.

11- Raheja, K. \& Maakar, S. K., 2014. A survey on different hybrid routing protocols of MANET. IJCSIT) 
International Journal of Computer Science and Information Technologies, pp. 5512-5516.

12- Renesse, R., Ghassemian, M., Friderikos, V. \& Aghvami, A. H., 2004. QoS enabled routing in mobile ad hoc networks. s.1., s.n., pp. 678-682.

13- Rohal, P., Dahiya, R. \& Dahiya, P., 2013. Study and analysis of throughput, delay and packet delivery ratio in MANET for topology based routing protocols (AODV, DSR and DSDV). international journal for advance research in engineering and technology, Volume 1, pp. 54-58.

14- Suradkar, S. S. \& Surve, A. R., 2014. A protocol for reducing routing overhead in mobile ad hoc networks. International Journal of Computer Science \& Engineering Technology, Volume 5.

15- Sylvia, D., Jothimohan, B. \& Rao, D. S., 2013. Study and Performance Evaluation of the Effect of Data Rate in Wireless Ad-Hoc Networks using IEEE 802. $11 \mathrm{~b}$ MAC protocol. International Journal of Computer Applications, Volume 84 https://doi.org/10.5120/14540-2615 